\title{
A New Restricted Full-rank Single-symbol Decodable Design for Four Transmit Antennas
}

\author{
Wei Liu ${ }^{1,2}$, Mathini Sellathurai ${ }^{1}$, Pei Xiao ${ }^{1}$, Jibo Wei $^{2}$
}

\begin{abstract}
Recently, a single-symbol decodable transmit strategy based on pre-processing at the transmitter has been introduced to decouple the Quasi-Orthogonal Space-time Block Codes (QOSTBC) with reduced complexity at the receiver [9]. Unfortunately, it does not achieve full diversity, thus suffers from significant performance loss. To tackle this problem, we propose a full diversity scheme with four transmit antennas in this letter. The proposed code is based on a class of restricted full-rank single-symbol decodable design (RFSDD) and has many similar characteristics to the Coordinate Interleaved Orthogonal Designs (CIODs), but has a lower peak-to-average ratio (PAR) .
\end{abstract}

Keywords- restricted full-rank single-symbol decodable design, coordinate interleaved orthogonal designs, full diversity, quasi-orthogonal space-time block code

\section{INTRODUCTION}

The Space-Time Block codes (STBCs) obtained from orthogonal designs (ODs) [1,2] provide a promising transmission scheme in multi-antenna systems due to their full diversity and single-symbol decodability (symbol-bysymbol decoding). However, it is proved in [2] that their symbol rates are upper bounded by $3 / 4$ when complex signal constellations and more than two transmit antennas are used. To increase the rate while preserving the full diversity, two classes of single-symbol decodable (SSD) STBCs have been proposed: (i) Coordinate Interleaved Orthogonal Designs (CIODs) [3] and (ii) Minimum-Decoding-Complexity (MDC) STBCs from Quasi-ODs (QODs) [4,5]. Recently, as an extension to ODs and CIODs that allow single-symboldecoding, B. Sundar Rajan, et al., proposed a so-called unrestricted full-rank single-symbol decodable design (UFSDD) and restricted full-rank single-symbol decodable design (RFSDD), respectively, in [6]. However, to the best of our knowledge, the CIODs or generalized CIODS (GCIODs) [6] are the only codes that were found satisfying restricted FSDD (RFSDD). In this letter, we obtain a new class of RFSDD other than CIODs or GCIODs.

Let us consider a $T \times N_{t}$ (we assume $T=N_{t}$ ) linear STBCs given by

$$
\mathbf{C}=\sum_{k=0}^{K-1} s_{k I} \mathbf{A}_{2 k}+s_{k Q} \mathbf{A}_{2 k+1}
$$

where $s_{k}=s_{k I}+j s_{k Q}, k=0, \ldots, K-1$, are the $K$ complex variables with $s_{k I}$ and $s_{k Q}$ denoting, respectively, the real and

1) The authors are with the Institute of Electronics, Communications, and Information Technology (ECIT), Queen's University of Belfast, Belfast BT3 9DT, U.K. (e-mail: \{wei.liu, m.sellathurai, pei.xiao\}@qub.ac.uk). 2) The authors are with the School of Electronic Science and Engineering, National University of Defense Technology, Changsha, 410073, China. (email: \{wliu_nudt,wjbhw\}@nudt.edu.cn). imaginary part of $s_{k}$ and $j=\sqrt{-1} . N_{t}$ denotes the number of transmit antennas, and $T$ is the number of time slots for one codeword. $\left\{\mathbf{A}_{k}\right\}_{k=0}^{2 K-1}$ is a set of $T \times N_{t}$ complex matrices called weight (dispersion) matrices of $\mathbf{C}$.

The following important conditions were introduced in [6] to classify single-symbol decodable STBCs:

$$
\begin{gathered}
\mathbf{A}_{k}^{H} \mathbf{A}_{l}+\mathbf{A}_{l}^{H} \mathbf{A}_{k}=0 \quad\left\{\begin{array}{l}
\forall l \neq k, k+1, \text { if } k \text { is even } \\
\forall l \neq k, k-1, \text { if } k \text { is odd }
\end{array}\right. \\
\mathbf{A}_{2 k}^{H} \mathbf{A}_{2 k+1}+\mathbf{A}_{2 k+1}^{H} \mathbf{A}_{2 k}=0,0 \leq k \leq K-1 \\
\operatorname{Rank}\left(\mathbf{A}_{k}^{H} \mathbf{A}_{k}\right)=N_{t}, 0 \leq k \leq K-1
\end{gathered}
$$

where $(\bullet)^{H}$ stands for the complex conjugate transpose of matrix (•), and expression (4) specifies $\mathbf{A}_{k}^{H} \mathbf{A}_{k}$ as a full-rank matrix for all $k$. A linear STBC is UFSDD if and only if all three conditions (2), (3) and (4) are satisfied. On the other hand, a linear STBC is RFSDD if and only if the following three conditions are satisfied:

i) The weight matrices satisfy conditions (2) and (3), but not satisfying (4);

ii) $\quad \mathbf{A}_{2 k}^{H} \mathbf{A}_{2 k}+\mathbf{A}_{2 k+1}^{H} \mathbf{A}_{2 k+1}$ is full-rank for all $0 \leq k \leq K-1$.

iii) The coordinate product distance (CPD) [6] of the signal set $\mathcal{A}$ is nonzero.

The single-symbol decodable STBCs presented in $[4,5,7,8]$ satisfy (2) and (4), but not (3). Thus, they are a new class of codes which differ from UFSDD and RFSDD.

By utilizing the fact that the eigenvectors of the equivalent channel are fixed and independent from the channel realizations, a single-symbol decodable transmit strategy based on pre-processing has been proposed in $[9,10]$ for the quasi-orthogonal space-time block codes (QOSTBC). For convenience, we call this scheme $\mathrm{SSD}^{[9]}$. However, the performance loss of $\mathrm{SSD}^{[9]}$ is significant as it does not achieve the full diversity order. In this letter, we derive a generic algebraic structure for systems with $N_{t}=4$ in order to gain a further insight into $\mathrm{SSD}^{[9]}$, and we discovered that the scheme $\operatorname{SSD}^{[9]}$ actually satisfies condition $i$ ). In light of this finding, we propose a full diversity design scheme for $\mathrm{SSD}^{[9]}$ in accordance with the condition ii) and iii), thus the performance is improved greatly in comparison to $\mathrm{SSD}^{[9]}$. It is a class of new RFSDD code as all conditions of RFSDD are satisfied. The proposed code shares many characteristics with CIOD but has a lower peak-to-average ratio (PAR) which is the major advantage of the proposed code over CIOD.

The remaining of this letter is organized as follows. In section II, we give the system model and a brief introduction to $\mathrm{SSD}^{[9]}$. The new RFSDD code is proposed in section III. The algebraic structure and coding gain of the proposed code 
are also analyzed in this section. Numerical results are presented in Section IV to demonstrate the effectiveness of the proposed codes. Finally, the conclusions are drawn in section V.

\section{SYSTEM MODEL}

We consider a MIMO system with $N_{t}=T=4$ and $N_{r}$ receive antennas. The system model is given by

$$
\mathbf{Y}=\mathbf{C H}+\mathbf{N},
$$

where $\mathbf{Y}=\left[y_{t, q}\right]_{T \times N_{r}}$ is the received signal matrix whose entry $y_{t, q}$ is the signal received at antenna $q$ at time $t$, where $t=1,2, \ldots, T$, and $q=1,2, \ldots, N_{r} ; \mathbf{N}=\left[n_{t, q}\right]_{T \times N_{r}}$ is the noise matrix; $\mathbf{C}=\left[c_{t, p}\right]_{T \times N_{t}}$ is the transmitted signal matrix whose entry $c_{t, p}$ is the signal transmitted at antenna $p$ and at time $t$, where $t=1,2, \ldots, T$, and $p=1,2, \ldots, N_{t} . \mathbf{H}=\left[h_{p, q}\right]_{N_{t} \times N_{r}}$ is the channel matrix whose entry $h_{p, q}$ is the channel coefficient from transmit antenna $p$ to receive antenna $q$. The entries of the matrices $\mathbf{H}$ and $\mathbf{N}$ are mutually independent, zero-mean, and complex Gaussian random variables. $h_{p, q}$ has unit variance and $n_{t, q}$ has variance $N_{t} / \rho$, where $\rho$ is the signal-to-noise ratio (SNR) per receive antenna. The channel is assumed to be flat fading and remains constant for a block of $T$ symbols and changes independently from block to block. It is further assumed that the transmitter has no channel state information (CSI) and the receiver has perfect CSI.

In the following, we focus on the $4 \times 4$ Quasi-Orthogonal Space-Time Block Codes (QOSTBCs) [11] and brief review the single-symbol decodable transmit strategy proposed in [9]. The codeword is given by

$$
\mathbf{C}=\left[\begin{array}{cccc}
x_{1} & x_{2} & x_{3} & x_{4} \\
x_{2}^{*} & -x_{1}^{*} & x_{4}^{*} & -x_{3}^{*} \\
x_{3} & -x_{4} & -x_{1} & x_{2} \\
x_{4}^{*} & x_{3}^{*} & -x_{2}^{*} & -x_{1}^{*}
\end{array}\right]
$$

which is a function of the vector $\mathbf{x}=\left[\begin{array}{llll}x_{1} & x_{2} & x_{3} & x_{4}\end{array}\right]^{T}$. After rearranging and complex-conjugating some rows of $\mathbf{Y},(5)$ can be reformed as

$$
\mathbf{y}^{\prime}=\mathbf{H}^{\prime} \mathbf{x}+\mathbf{n}^{\prime},
$$

where $\mathbf{y}^{\prime}=\left[\left(\mathbf{y}_{1}\right)^{T},\left(\mathbf{y}_{2}\right)^{T}, \ldots,\left(\mathbf{y}_{N_{r}}\right)^{T}\right]^{T}, \mathbf{H}^{\prime}=\left[\left(\mathbf{H}_{1}\right)^{T},\left(\mathbf{H}_{2}\right)^{T}, \ldots,\left(\mathbf{H}_{N_{r}}\right)^{T}\right]^{T}$, and $\quad \mathbf{n}^{\prime}=\left[\left(\mathbf{n}_{1}\right)^{T},\left(\mathbf{n}_{2}\right)^{T}, \ldots,\left(\mathbf{n}_{N_{r}}\right)^{T}\right]^{T}$

$\mathbf{n}_{q}=\left[\begin{array}{llll}n_{1, q} & n_{2, q}^{*} & n_{3, q} & n_{4, q}^{*}\end{array}\right]^{T}, \quad q=1,2, \ldots, N_{r} \cdot(\bullet)^{T}$ denotes the transpose of matrix $(\bullet)$, and $(\bullet)^{*}$ denotes the conjugate of a complex scalar. $\mathbf{y}_{q}, \mathbf{H}_{q}$ are given respectively as $\mathbf{y}_{q}=\left[\begin{array}{llll}y_{1, q} & y_{2, q}^{*} & y_{3, q} & y_{4, q}^{*}\end{array}\right]^{T}, \quad \mathbf{H}_{q}=\left[\begin{array}{cccc}h_{1, q} & h_{2, q} & h_{3, q} & h_{4, q} \\ -h_{2, q}^{*} & h_{1, q}^{*} & -h_{4, q}^{*} & h_{3, q}^{*} \\ -h_{3, q} & h_{4, q} & h_{1, q} & -h_{2, q} \\ -h_{4, q}^{*} & -h_{3, q}^{*} & h_{2, q}^{*} & h_{1, q}^{*}\end{array}\right]$.

We get the equivalent channel matrix $\mathbf{H}^{H^{H}} \mathbf{H}^{\prime}$ as

$$
\mathbf{H}^{H} \mathbf{H}^{\prime}=\left[\begin{array}{cccc}
\alpha_{1} & 0 & \alpha_{2} j & 0 \\
0 & \alpha_{1} & 0 & -\alpha_{2} j \\
-\alpha_{2} j & 0 & \alpha_{1} & 0 \\
0 & \alpha_{2} j & 0 & \alpha_{1}
\end{array}\right]
$$

where $\alpha_{1}$ and $\alpha_{2}$ are defined as

$$
\alpha_{1}=\sum_{q=1}^{N_{r}} \sum_{p=1}^{4}\left|h_{p, q}\right|^{2} ; \alpha_{2}=\sum_{q=1}^{N_{r}} 2 \operatorname{Im}\left(h_{1, q}^{*} h_{3, q}+h_{4, q}^{*} h_{2, q}\right) .
$$

We use the $\operatorname{Re}(\bullet)$ and $\operatorname{Im}(\bullet)$ to denote the real and imaginary parts of a complex scalar, respectively.

The symmetric matrix in (8) has the following singular value decomposition (SVD) as

$$
\mathbf{H}^{H} \mathbf{H}^{\prime}=\mathbf{V D D V}^{H},
$$

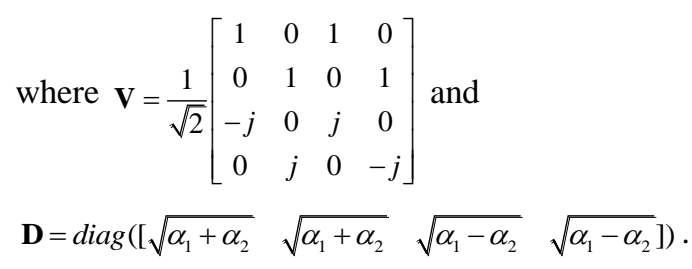

An important characteristic is that the matrix $\mathbf{V}$ is a constant unitary matrix for arbitrary channel realizations. If we choose $\mathbf{V}$ as a pre-processing matrix in the transmitter, the vector $\mathbf{x}$ in (7) can be rewritten as

$$
\mathbf{x}=\mathbf{V s},
$$

where $\mathbf{s}=\left[\begin{array}{llll}s_{1} & s_{2} & s_{3} & s_{4}\end{array}\right]^{T}$, and $s_{1}, s_{2}, s_{3}, s_{4} \in \mathcal{A}, \mathcal{A}$ is a complex signal set with unit average power. Combining expressions (7), (10) and (11) results in a completely decoupled model as follows

$$
\mathbf{y}^{\prime \prime}=\mathbf{D s}+\mathbf{w}=\mathbf{D}^{-1} \mathbf{V}^{H} \mathbf{H}^{H} \mathbf{y}^{\prime},
$$

Where $\mathbf{w}$ is the noise vector, whose entries are mutually i.i.d. complex Gaussian random variables with zero-mean and iden tical variance.

\section{PROPOSED RFSDD CODE}

A single-symbol decodable transmit strategy SSD has been derived in [9] based on the completely decoupled model expressed by (12). Unfortunately, $\operatorname{SSD}^{[9]}$ does not achieve the full diversity provided by the MIMO channel, which will be proven below.

\section{A. Analysis of SSD $^{[9]}$}

Substituting (11) into (6) and combining with (1), we get the weight matrices of $\operatorname{SSD}^{[9]}$ with $K=4$ as follows:

$$
\begin{array}{rlrl}
\mathbf{A}_{0} & =\frac{1}{\sqrt{2}}\left[\begin{array}{cccc}
1 & 0 & -j & 0 \\
0 & -1 & 0 & -j \\
-j & 0 & -1 & 0 \\
0 & j & 0 & -1
\end{array}\right] & \mathbf{A}_{1}=\frac{1}{\sqrt{2}}\left[\begin{array}{cccc}
j & 0 & 1 & 0 \\
0 & j & 0 & -1 \\
1 & 0 & -j & 0 \\
0 & 1 & 0 & j
\end{array}\right] \\
\mathbf{A}_{2}=\frac{1}{\sqrt{2}}\left[\begin{array}{cccc}
0 & 1 & 0 & j \\
1 & 0 & -j & 0 \\
0 & -j & 0 & 1 \\
-j & 0 & -1 & 0
\end{array}\right] & \mathbf{A}_{3}=\frac{1}{\sqrt{2}}\left[\begin{array}{cccc}
0 & j & 0 & -1 \\
-j & 0 & -1 & 0 \\
0 & 1 & 0 & j \\
-1 & 0 & j & 0
\end{array}\right]
\end{array}
$$




$$
\begin{array}{rlrl}
\mathbf{A}_{4} & =\frac{1}{\sqrt{2}}\left[\begin{array}{cccc}
1 & 0 & j & 0 \\
0 & -1 & 0 & j \\
j & 0 & -1 & 0 \\
0 & -j & 0 & -1
\end{array}\right] & \mathbf{A}_{5}=\frac{1}{\sqrt{2}}\left[\begin{array}{cccc}
j & 0 & -1 & 0 \\
0 & j & 0 & 1 \\
-1 & 0 & -j & 0 \\
0 & -1 & 0 & j
\end{array}\right] \\
\mathbf{A}_{6}=\frac{1}{\sqrt{2}}\left[\begin{array}{cccc}
0 & 1 & 0 & -j \\
1 & 0 & j & 0 \\
0 & j & 0 & 1 \\
j & 0 & -1 & 0
\end{array}\right] & \mathbf{A}_{7}=\frac{1}{\sqrt{2}}\left[\begin{array}{cccc}
0 & j & 0 & 1 \\
-j & 0 & 1 & 0 \\
0 & -1 & 0 & j \\
1 & 0 & j & 0
\end{array}\right]
\end{array}
$$

It is easy to verify that all the weight matrices satisfy condition $i$ ) but not conditions ii) and iii), so that only partial diversity can be obtained no matter how we design CPD. Next, we present a re-grouping scheme to make the code satisfying condition ii).

\section{B. Re-grouping scheme to satisfy condition ii)}

We group all the weight matrices $\mathbf{A}_{0}, \mathbf{A}_{1}, \mathbf{A}_{2}, \mathbf{A}_{3}, \mathbf{A}_{4}$, $\mathbf{A}_{5}, \mathbf{A}_{6}, \mathbf{A}_{7}$ into $K=4$ groups as follows: $g_{k}=\left\{\mathbf{A}_{p}, \mathbf{A}_{q}\right\}$, where $k=0,1,2,3$ and $p, q=0,1, \ldots, 7, p \neq q$. The $\mathbf{A}_{p}$ and $\mathbf{A}_{q}$ in $g_{k}$ are the coefficient matrices of $s_{k l}$ and $s_{k q}$, respectively, where $s_{k l}+j s_{k Q} \in \mathcal{A}, k=0,1,2,3$. For example, the expression (1) shows that the groups are $g_{0}=\left\{\mathbf{A}_{0}, \mathbf{A}_{1}\right\}, g_{1}=\left\{\mathbf{A}_{2}, \mathbf{A}_{3}\right\}$, $g_{2}=\left\{\mathbf{A}_{4}, \mathbf{A}_{5}\right\}, g_{3}=\left\{\mathbf{A}_{6}, \mathbf{A}_{7}\right\}$.

Another interpretation of condition $\mathrm{ii}$ ) is that for any group $g_{k}=\left\{\mathbf{A}_{p}, \mathbf{A}_{q}\right\}, \quad k=0,1,2,3$ and $p, q=0,1, \ldots, 7, \quad p \neq q$, it is necessary for the matrix $\mathbf{A}_{p}^{H} \mathbf{A}_{p}+\mathbf{A}_{q}^{H} \mathbf{A}_{q}$ to have full-rank. Next, we will re-group the weight matrices to derive a new code that satisfies condition ii).

After examining all possible groups for weight matrices, we obtain

$$
\operatorname{Rank}\left(\mathbf{A}_{p}^{H} \mathbf{A}_{p}+\mathbf{A}_{q}^{H} \mathbf{A}_{q}\right)=\left\{\begin{array}{lrll}
2, & p, q \in N_{1} & \text { or } & p, q \in N_{2} \\
4, & p \in N_{1}, q \in N_{2} & \text { or } & p \in N_{2}, q \in N_{1}
\end{array}\right.
$$

where $N_{1}=\{0,1,2,3\}$ and $N_{2}=\{4,5,6,7\}$. In Table 1 ,

we denote " $\Delta$ " as the two weight matrices that satisfy (15).

\section{Table 1: Groups of weight matrices}

\begin{tabular}{c|cccccccc} 
& $\mathbf{A}_{0}$ & $\mathbf{A}_{1}$ & $\mathbf{A}_{2}$ & $\mathbf{A}_{3}$ & $\mathbf{A}_{4}$ & $\mathbf{A}_{5}$ & $\mathbf{A}_{6}$ & $\mathbf{A}_{7}$ \\
\hline $\mathbf{A}_{0}$ & & & & & $\Delta$ & $\Delta$ & $\Delta$ & $\Delta$ \\
$\mathbf{A}_{1}$ & & & & & $\Delta$ & $\Delta$ & $\Delta$ & $\Delta$ \\
$\mathbf{A}_{2}$ & & & & & $\Delta$ & $\Delta$ & $\Delta$ & $\Delta$ \\
$\mathbf{A}_{3}$ & & & & & $\Delta$ & $\Delta$ & $\Delta$ & $\Delta$
\end{tabular}

\begin{tabular}{l|cccc}
$\mathbf{A}_{4}$ & $\Delta$ & $\Delta$ & $\Delta$ & $\Delta$ \\
$\mathbf{A}_{5}$ & $\Delta$ & $\Delta$ & $\Delta$ & $\Delta$ \\
$\mathbf{A}_{6}$ & $\Delta$ & $\Delta$ & $\Delta$ & $\Delta$ \\
$\mathbf{A}_{7}$ & $\Delta$ & $\Delta$ & $\Delta$ & $\Delta$
\end{tabular}

It is noteworthy that there are many grouping patterns to satisfy condition ii). As an example, we choose $g_{0}=\left\{\mathbf{A}_{7}, \mathbf{A}_{0}\right\}$, $g_{1}=\left\{\mathbf{A}_{5}, \mathbf{A}_{2}\right\}, g_{2}=\left\{\mathbf{A}_{3}, \mathbf{A}_{4}\right\}, g_{3}=\left\{\mathbf{A}_{1}, \mathbf{A}_{6}\right\}$ shown as “ $\Delta$ " in

Table 1. The new code is obtained as

$\mathbf{C}^{\prime}=s_{0 I} \mathbf{A}_{7}+s_{0 Q} \mathbf{A}_{0}+s_{1 I} \mathbf{A}_{5}+s_{1 Q} \mathbf{A}_{2}+s_{2 I} \mathbf{A}_{3}+s_{2 Q} \mathbf{A}_{4}+s_{3 I} \mathbf{A}_{1}+s_{3 Q} \mathbf{A}_{6}$

It is easy to prove that the re-grouping has no effect on condition i). Combining (13) and (16), the code can be rewritten as (17) at the top of next page, which is an interleaved version of $\mathrm{SSD}^{[9]}$ and satisfies both condition i) and $i$ ).

\section{Optimal constellation design}

Condition iii) can always be satisfied by rotating a given signal set $\mathcal{A}$. In order to design an optimal rotation angle to maximize the coding gain, we first derive the determinant expression for the codeword distance matrix, from which the coding gain can be completely determined. Based on the codeword definition in (16), we let

$\mathbf{C}^{\prime}=\mathbf{C}^{\prime}\left(s_{0}, s_{1}, s_{2}, s_{3}\right)$ and $\hat{\mathbf{C}^{\prime}}=\mathbf{C}^{\prime}\left(\hat{s_{0}}, \hat{s_{1}}, \hat{s_{2}}, \hat{s_{3}}\right)$,

$\Delta \mathbf{C}=\mathbf{C}^{\prime}-\hat{\mathbf{C}}^{\prime} \neq 0$. Combining (13) and (16), we obtain

$$
\begin{aligned}
\operatorname{det}\left(\Delta \mathbf{C}^{H} \Delta \mathbf{C}\right) & =\left(4\left(\Delta s_{0 I}{ }^{2}+\Delta s_{1 I}{ }^{2}+\Delta s_{2 Q}{ }^{2}+\Delta s_{3 Q}{ }^{2}\right)\right. \\
& \left.\bullet\left(\Delta s_{0 Q}{ }^{2}+\Delta s_{1 Q}{ }^{2}+\Delta s_{2 I}{ }^{2}+\Delta s_{3 I}{ }^{2}\right)\right)^{2}
\end{aligned}
$$

where $\quad \Delta s_{k l}=s_{k I}-\hat{s_{k I}} \quad, \quad \Delta s_{k Q}=s_{k Q}-\hat{s_{k Q}} \quad, \quad s_{k}=s_{k I}+j s_{k Q} \quad$, $\hat{s_{k}}=\hat{s_{k l}}+j \hat{s_{k Q}}$, and at least one $s_{k}$ differs from $\hat{s_{k}}, k=0,1,2,3$

To obtain the minimum of determinant expression in (18), without loss of generality, we assume $s_{k} \neq \hat{s_{k}}$ only for $k=0$. Then (18) can be rewritten as

$$
\operatorname{det}\left(\Delta \mathbf{C}^{H} \Delta \mathbf{C}\right)=\left(4 \Delta s_{0 I}{ }^{2} \Delta s_{0 Q}{ }^{2}\right)^{2} .
$$

Consequently, maximization of the coding gain is equivalent to maximization of the CPD of signal set $\mathcal{A}$, which is similar to CIODs. The optimal angle of rotation for CIODs with all different constellations is directly applicable to the code in (17). As an example, the optimal angle of rotation for square QAM is $\theta_{\text {opt }}=\arctan (2) / 2=31.7175^{\circ}$.

Considering the CIOD shown in (85) of [6], we can derive its determinant expression for codeword distance matrix as

$$
\begin{aligned}
\operatorname{det}\left(\Delta \mathbf{C}^{H} \Delta \mathbf{C}\right) & =\left(\left(\Delta s_{0 I}{ }^{2}+\Delta{s_{1 I}}^{2}+\Delta s_{2 Q}{ }^{2}+\Delta s_{3 Q}{ }^{2}\right)\right. \\
& \left.\bullet\left(\Delta s_{0 Q}{ }^{2}+\Delta s_{1 Q}{ }^{2}+\Delta s_{2 I}{ }^{2}+\Delta s_{3 I}{ }^{2}\right)\right)^{2}
\end{aligned}
$$

which differs from (18) only in one coefficient. However the energy in (20) is double of that in (18) because only half of transmit antennas are utilized for CIOD. Therefore, the proposed code has the same coding gain as CIOD.

\section{ML Decoding}

It can be shown that the ML decoding metric can be calculate as the sum $f_{0}\left(s_{0}\right)+f_{1}\left(s_{1}\right)+f_{2}\left(s_{2}\right)+f_{3}\left(s_{3}\right)$, where

$$
\begin{aligned}
& f_{0}\left(s_{0}\right)=\left(y^{\prime \prime}{ }_{3 Q}-d_{2} s_{0 I}\right)^{2}+\left(y^{\prime \prime}{ }_{0 I}-d_{1} s_{0 Q}\right)^{2} \\
& f_{1}\left(s_{1}\right)=\left(y^{\prime \prime}{ }_{2 Q}-d_{2} s_{1 I}\right)^{2}+\left(y^{\prime \prime}{ }_{1 I}-d_{1} s_{1 Q}\right)^{2} \\
& f_{2}\left(s_{2}\right)=\left(y^{\prime \prime}{ }_{1 Q}-d_{1} s_{2 I}\right)^{2}+\left(y^{\prime \prime}{ }_{2 I}-d_{2} s_{2 Q}\right)^{2} \\
& f_{3}\left(s_{3}\right)=\left(y^{\prime \prime}{ }_{0 Q}-d_{1} s_{3 I}\right)^{2}+\left(y^{\prime \prime}{ }_{3 I}-d_{2} s_{3 Q}\right)^{2},
\end{aligned}
$$

where $d_{1}=\sqrt{\alpha_{1}+\alpha_{2}}, d_{2}=\sqrt{\alpha_{1}-\alpha_{2}}$, and

$\left[\begin{array}{l}\operatorname{Re}\left(y^{\prime \prime}\right) \\ \operatorname{Im}\left(y^{\prime \prime}\right)\end{array}\right]=\left[\begin{array}{llllllll}y^{\prime \prime}{ }_{0 I} & y^{\prime \prime}{ }_{1 I} & y^{\prime \prime}{ }_{2 I} & y^{\prime \prime}{ }_{3 I} & y^{\prime \prime}{ }_{0 Q} & y^{\prime \prime}{ }_{1 Q} & y^{\prime \prime}{ }_{2 Q} & y^{\prime \prime}{ }_{3 Q}\end{array}\right]^{T}$. 


$$
\mathbf{C}^{\prime}=\frac{1}{\sqrt{2}}\left[\begin{array}{cccc}
s_{0 Q}+s_{2 Q}+j s_{3 I}+j s_{1 I} & s_{1 Q}+s_{3 Q}+j s_{2 I}+j s_{0 I} & s_{3 I}-s_{1 I}-j s_{0 Q}+j s_{2 Q} & -s_{2 I}+s_{0 I}+j s_{1 Q}-j s_{3 Q} \\
s_{1 Q}+s_{3 Q}-j s_{2 I}-j s_{0 I} & -s_{0 Q}-s_{2 Q}+j s_{3 I}+j s_{1 I} & -s_{2 I}+s_{0 I}-j s_{1 Q}+j s_{3 Q} & -s_{3 I}+s_{1 I}-j s_{0 Q}+j s_{2 Q} \\
s_{3 I}-s_{1 I}-j s_{0 Q}+j s_{2 Q} & s_{2 I}-s_{0 I}-j s_{1 Q}+j s_{3 Q} & -s_{0 Q}-s_{2 Q}-j s_{3 I}-j s_{1 I} & s_{1 Q}+s_{3 Q}+j s_{2 I}+j s_{0 I} \\
-s_{2 I}+s_{0 I}-j s_{1 Q}+j s_{3 Q} & s_{3 I}-s_{1 I}+j s_{0 Q}-j s_{2 Q} & -s_{1 Q}-s_{3 Q}+j s_{2 I}+j s_{0 I} & -s_{0 Q}-s_{2 Q}+j s_{3 I}+j s_{1 I}
\end{array}\right]
$$

\section{NUMERICAL RESULTS}

The simulation results are presented in this section for different codes with four transmit antennas. All the codes employ QPSK constellation. The rotation angle is set to $31.7175^{\circ}$ for both CIOD and the proposed code. The biterror-rate (BER) performance for $\mathrm{SSD}^{[9]}, \mathrm{CIOD}$, and the proposed code are shown in Fig. 1. It is observed that the proposed design improve the performance of the original $\mathrm{SSD}^{[9]}$ significantly (about $5 \mathrm{~dB}$ performance gain can be obtained at $\mathrm{BER}=10^{-4}$ with one receive antenna). The proposed RFSDD code has identical performance to CIOD. Similar observations hold for the case with two receive antennas.

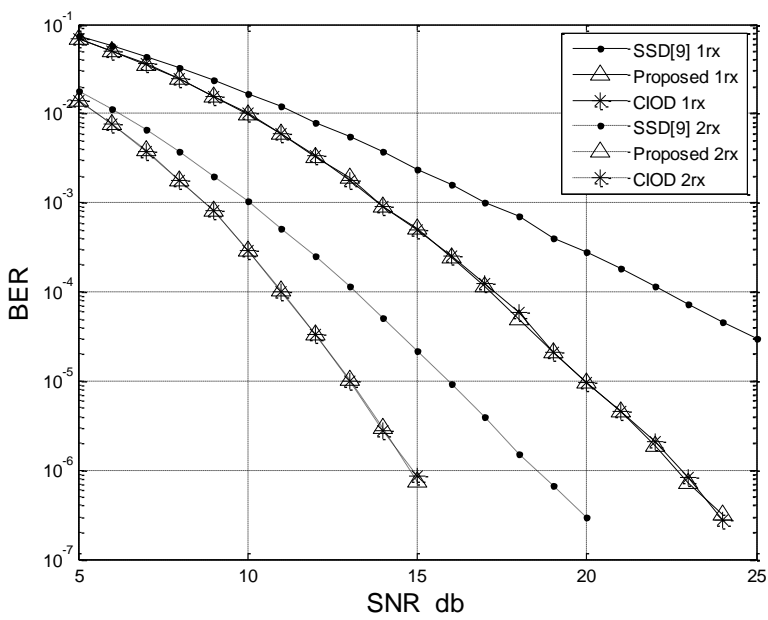

Fig. 1 Simulation results of $\mathrm{SSD}^{[9]}$, CIOD, and proposed code for four transmit antennas

\section{CONCLUSION}

The proposed code in this letter is a full diversity version of $\mathrm{SSD}^{[9]}$. It is a new RFSDD and its coding gain and optimal angle of constellation rotation are the same as CIOD. Simulation results show that the proposed code has a more rapid $\mathrm{BER}$ slope than $\mathrm{SSD}^{[9]}$ and achieve the identical performance to CIOD. However, the proposed code has more dispersive power and lower peak-to-average ratio (PAR) than CIOD since half of the antennas are idle [6] in the latter case.

\section{REFERENCES}

[1] S. M. Alamouti,"A simple transmit diversity technique for wireless communications”, IEEE J. Select. Areas Commun. 1998, 16(8): 14511458

[2] V. Tarokh, H. Jafarkhani, and A. R. Calderbank, "Space-time block codes from orthogonal designs", IEEE Trans. Inform. Theory. 1999, 45(5): 1456-1467.

[3] M. Zafar A. Khan and B. S. Rajan, "Space-Time Block Codes from Co-ordinate Interleaved Orthogonal Designs," Proc: IEEE International Symposium on Information Theory, (ISIT 2002), Lausanne, Switzerland, June 30-July 5, 2002, p.275.

[4] C. Yuen, Y. L. Guan and T. T. Tjhung, "Quasi-orthogonal STBC with minimum decoding complexity," IEEE Trans. Wireless Commun., vol. 4, no. 5, pp.2089-2094. Sep. 2005.

[5] C. Yuen; Y. L. Guan; T. T. Tjhung, "Quasi-Orthogonal STBC with Minimum Decoding Complexity: Further Results", Proc. IEEE WCNC New Orleans, LA, USA, March 2005. Volume 1, Page(s):483 -488.

[6] M. Zafar A. Khan and B. S. Rajan,"Single-Symbol MaximumLikelihood Decodable Linear STBCs," IEEE Trans. Inform. Theory, vol. 52, pp. 2062-2091, May 2006.

[7] S. Karmakar and B. S. Rajan, "Minimum-Decoding-Complexity, Maximum-rate Space-Time Block Codes from Clifford Algebras,' Proc. IEEE ISIT 2006, Seattle, USA, July 9-14, 2006.

[8] G. Yuen, Y. L. Guan and T. T. Tjhung, "Optimizing quasi-orthogonal STBC through group-constrained linear transformation," IET Commun., 2007, 1, (3), pp.373-381.

[9] A. Sezgin and T. J. Oechtering, "Complete characterization of the equivalent MIMO Channel for quasi-orthogonal space-time codes", submitted to IEEE Trans. on Info. Theory. Also available at http://www.stanford.edu/ sezgin/Publications, 2004.

[10] A. Szezgin. "Space-Time Codes for MIMO Systems: QuasiOrthogonal Design and Concatenation". pp.39-40. PhD thesis, Technical University Berlin, Germany, June 2005. Available at http://www.stanford.edu/ sezgin/Publications.

[11] C. B. Papadias and G. J. Foschini, "Capacity-approaching space-time codes for systems employing four transmitter antennas," IEEE Trans. Inform. Theory, vol. 49, pp. 726-732, Mar. 2003. 